\title{
Synergistic antidigestion effect of Lactobacillus rhamnosus and bovine colostrums in simulated gastrointestinal tract (in vitro)
}

Hua Wei • Yang Xu • Yonghua Xiong • Feng Xu •

Gengpin Liu

Published online: 3 April 2007

(C) Springer-Verlag 2007

\section{Appl Microbiol Biotechnol}

DOI: $10.1007 / \mathrm{s} 00253-007-0861-8$

In the original version of this article the author's names were rendered incorrectly: the family names and given names were reversed. The names are shown correctly here.

The online version of the original article can be found at http://dx.doi. org/10.1007/s00253-007-0861-8.

H. Wei $(\bowtie) \cdot Y$. Xu $\cdot$ Y. Xiong $\cdot$ F. Xu $\cdot$ G. Liu

Jiangxi-OAI Joint Research Institute, Nanchang University,

Nanchang, Jiangxi 330047, People's Republic of China

e-mail: weihua114@hotmail.com

H. Wei $\cdot$ Y. Xu $\cdot$ Y. Xiong

The Key Laboratory of Food Science of MOE,

Nanchang University,

Nanchang, Jiangxi 330047, People's Republic of China 